\title{
BMJ Open Oral cannabinoids in people living with HIV on effective antiretroviral therapy: CTN PT028 - study protocol for a pilot randomised trial to assess safety, tolerability and effect on immune activation
}

Cecilia T Costiniuk, ${ }^{1,2}$ Zahra Saneei, ${ }^{1}$ Jean-Pierre Routy, ${ }^{1,2}$ Shari Margolese, ${ }^{3}$ Enrico Mandarino, ${ }^{3,4}$ Joel Singer, ${ }^{3,5}$ Bertrand Lebouché, ${ }^{1,2,6}$ Joseph Cox, ${ }^{1,2,6}$ Jason Szabo, , ${ }^{1,6}$ Marie-Josée Brouillette, ${ }^{1,2,7}$ Marina B Klein, ${ }^{1,2}$ Nicolas Chomont, ${ }^{8}$ Mohammad-Ali Jenabian ${ }^{9}$

To cite: Costiniuk CT, Saneei Z, Routy J-P, et al. Oral cannabinoids in people living with HIV on effective antiretroviral therapy: CTN PT028 - study protocol for a pilot randomised trial to assess safety, tolerability and effect on immune activation. BMJ Open 2019;9:e024793. doi:10.1136/ bmjopen-2018-024793

\section{- Prepublication history for} this paper is available online. To view these files, please visit the journal online (http://dx.doi. org/10.1136/bmjopen-2018024793).

Received 15 June 2018 Revised 17 0ctober 2018 Accepted 8 November 2018

Check for updates

(C) Author(s) (or their employer(s)) 2019. Re-use permitted under CC BY-NC. No commercial re-use. See rights and permissions. Published by BMJ.

For numbered affiliations see end of article.

Correspondence to Dr Cecilia T Costiniuk; cecilia.costiniuk@mcgill.ca

\begin{abstract}
Introduction Despite antiretroviral therapy (ART), people living with HIV have higher rates of non-infectious chronic diseases. These conditions are driven by relatively high levels of inflammation persisting on ART compared with uninfected individuals. Chronic inflammation also contributes to HIV persistence during ART. Cannabis when taken orally may represent a way to reduce inflammation and strengthen immune responses. Before planning large interventional studies, it is important to ensure that cannabis taken orally is safe and well tolerated in people living with HIV. We propose to conduct a pilot randomised trial to examine the safety and tolerability of cannabis oils containing tetrahydrocannabinol (THC) and cannabidiol (CBD) consumed orally in people living with HIV. We will also measure inflammatory markers, markers of HIV persistence in peripheral blood cells and changes in the gastrointestinal microbiome.
\end{abstract}

Methods and analysis Twenty-six people living with HIV having undetectable viral load for at least 3 years will be randomised to receive TN-TC11LM (THC:CBD in 1:1 ratio) or TN-TC19LM (THC:CBD in 1:9 ratio) capsules daily for 12 weeks. Safety and tolerability of these capsules will be assessed through haematological, hepatic and renal blood tests, face-to-face interviews and questionnaires. Proportions of participants without any signs of significant toxicity (grades 0-2 scores on the WHO toxicity scale) and who complete the study, as well as scores on quality of life and mood will be examined using descriptive statistics. The effects on inflammatory markers, markers of peripheral blood reservoir size and effect on the composition of the gastrointestinal microbiome will be assessed before and after study completion.

Ethics and dissemination This study has been approved by the Research Institute of the McGill University Health Centre. A Data Safety Monitor will review safety information at regular intervals. The final manuscript will be submitted to an open-access journal within 6 months of study completion.
Strengths and limitations of this study

Randomised clinical trial design involving oral consumption of capsules containing two different ratios of cannabinoids.

- The capsules used will contain both $\Delta 9$ tetrahydrocannabinol and cannabidiol, thus improving tolerability.

- The use of oral capsules containing precise amounts of cannabinoids, rather than smoked cannabis, ensures more predictable dosage administration and avoids the harmful pulmonary effects associated with smoking.

- Effects of the intervention on quality of life, cognition and mood in addition to biological outcomes are being examined.

- The number of participants should provide insight into the degree of variability for continuous outcomes and should guide future sample size calculations for larger studies.

Trial registration number NCT03550352.

\section{BACKGROUND}

Despite antiretroviral therapy (ART), persistent immune activation is associated with increased risk of non-opportunistic complications in people living with HIV such as cardiovascular, pulmonary, renal and hepatic events. ${ }^{12}$ HIV pathogenesis and persistence appear to be related to chronic inflammation and immune activation, ${ }^{3}$ driven by microbial translocation of bacterial products across the gut mucosa. ${ }^{4-6}$ Even when ART is initiated in the primary or early phase 
of HIV, gut integrity is not fully restored. ${ }^{7}$ Furthermore, there is recent evidence to suggest that inflammatory features of the enteric microbiota, and not just increased permeability alone, is driving chronic inflammation in people living with HIV. Indeed, some studies have shown correlations between specific enteric bacteria and immune activation markers in gut and blood. ${ }^{89}$ Persistent immune activation may also contribute to the persistence of HIV during ART. ${ }^{10-15}$ HIV reservoirs are the reason why HIV remains an incurable infection. Although HIV may also persist in myeloid cells, $\mathrm{CD} 4+\mathrm{T}$ cells are the best characterised and the most abundant reservoirs. ${ }^{16-18}$

Attenuation of immune activation and levels of inflammation may portend several therapeutic benefits to people living with HIV, and novel strategies are needed to achieve this goal. Having both anti-inflammatory and antifibrotic properties, ${ }^{19}$ cannabis may represent a feasible method to reduce immune activation and enhance immune profile. When consumed orally, notably soluble in oil, it may also temper inflammation at the gastrointestinal mucosa. ${ }^{20}$ This, in turn, may hasten the progression of non-opportunistic complications associated with HIV. Furthermore, cannabis use, mainly through smoking, is already extremely common among people living with HIV (PLWHIV). A study conducted by our team involving 1072 participants in the Canadian Co-infection Cohort Study (CTN 222), a multicentred longitudinal study of HIV-hepatitis C coinfected individuals, revealed that $53 \%$ of individuals had smoked marijuana in the past 6 months. ${ }^{21}$ A clearer understanding of the safety, tolerability and feasibility of using cannabis in a clinical trial is an important first step before designing interventional studies to ameliorate specific conditions of importance to people living with HIV. Furthermore, as cannabis use will be legalised in Canada in July 2018, understanding its safety profile is very important.

\section{Pharmacology and medical properties of cannabis}

Cannabinoids, found in the hemp plant Cannabis sativa, have been recognised for centuries for their analgesic, anticonvulsant, bronchodilatory, sedative, hypnotic and antispasmodic properties. ${ }^{22} 23$ Their biological activity is conferred by cannabinoid receptors $\mathrm{CB} 1$ and $\mathrm{CB} 2$ through activation of heterodimeric G-proteins that function as signalling and regulatory proteins to operate or modulate intracellular signalling pathways. ${ }^{24} 25$ While CB1 receptors are expressed predominantly in the central nervous system, they are also found in the lung, liver and kidneys. The endocannabinoid system also plays a key role in the gastrointestinal tract's neural and molecular control mechanisms. Indeed, the endocannabinoid system plays a role in normal physiological functions of the gastrointestinal tract including motility, gut-brain mediated fat intake, hunger signalling, inflammation and gut permeability. ${ }^{26}$ Furthermore, there is some evidence for and interactions between the endocannaboid system and the gut microbiota. ${ }^{26}$ In lean mice who received 4 weeks of a cannabinoid-receptor agonist HU-210, plasma lipopolysaccharides (LPS) were significantly increased. ${ }^{26} 27$ When obese mice with disrupted gastrointestinal mucosa were treated with rimonabant, an inverse CB1 agonist, for nearly 2 weeks, reductions in plasma LPS were observed. ${ }^{26}{ }^{28}$ Furthermore, improvements in localisation of tight junction proteins, occluding and zonula occludens-1 were measured, suggestive of improvement in endothelial barrier function. ${ }^{26} 28$

The presence of cannabinoid receptors in the central nervous system accounts for the psychoactive effects of cannabis. ${ }^{24}{ }^{25}$ In contrast, CB2 receptors are abundant on immune cells including $\mathrm{T}$ and B cells, natural killer cells, monocytes and neutrophils as well as the liver. ${ }^{24} 29$ Two primary active constituents found in hemp plants include $\Delta$ 9-tetrahydrocannabinol (THC) and cannabidiol (CBD). $\triangle 9$-THC is a partial agonist at both CB1 and CB2, ${ }^{3} 3031$ while CBD is thought to mediate its effects through a variety of signalling mechanisms and act as a negative allosteric modulator at $\mathrm{CB} 1 .^{32} 33$

Cannabis is perhaps best known for its effect on stimulating appetite via $\mathrm{CB} 1$ receptor activity and was historically used for this purpose in AIDS wasting syndrome. A variety of randomised controlled trials have shown that smoked cannabis is efficacious in the management of chronic pain, including in painful HIV-associated sensory neuropathy. ${ }^{34-36}$ In animal models, $\triangle 9$-THC prevents atherosclerosis by inhibiting macrophage migration into atheromas through CB2 activation. ${ }^{37} 38$ Endocannabinoids may also play a role in liver disease, where CB1 receptors are present in endothelial cells and hepatocytes and CB2 receptors are distributed in Kupffer cells. ${ }^{39} 40$ CB1 receptors have an important role in non-alcoholic fatty liver disease and alcoholic liver disease, while anti-inflammatory action of CB2 receptors can be useful in inflammatory liver disease. CB2 receptors show antifibrinogenic properties and administration of its agonists in fibrotic rats resulted in improvement in liver fibrosis, decreased inflammation and increased apoptosis of hepatic myofibroblasts. ${ }^{40-44}$

\section{CANNABIS, THE IMMUNE SYSTEM AND HIV In vitro studies}

Cannabinoids have been shown to inhibit productive HIV infection in primary human T cells, and a CB2 antagonist blocked this effect. ${ }^{45}$ Interference with the signal transduction of chemokine receptor CXCR4 is thought to lead to reduced F-actin accumulation. This in turn prevents movement of viral preintegration complexes to the nucleus. It has also been postulated that CB2 agonists may inhibit $\mathrm{T}$ cell activation induced by anti-CD3/anti-CD28. ${ }^{45}$ Along with reduced HIV production, immunological effects of cannabinoids include induction of immunosuppressive cytokines including interleukin (IL)-10, tumour growth factor (TGF) $-\beta$ and inhibition of IL-2, which stimulates $\mathrm{T}$ cell division and expansion. They have also been shown to decrease adhesion capacity of leukocytes to 
extravasate into sites of inflammation. ${ }^{46-48}$ Apoptosis and induction of Treg may potentially add to the pathways of cannabinoid-mediated immunosuppression. ${ }^{49}$

\section{Animal studies}

Mice treated with $\triangle 9$-THC experience persistent inhibition of IL-2 production even 7 days after treatment. ${ }^{50}$ Rhesus macaques who were administered $\triangle 9$-THC for 28 days prior to simian immunodeficiency virus (SIV) inoculation had reduced mortality and reduced SIV viral load in the cerebrospinal fluid and plasma. In another study involving macaques infected with SIV for 17 months of chronic THC administration, ${ }^{51} \Delta 9$-THC resulted in trends towards a decreased viral load. The lack of statistically significance in some parameters can be attributed to the small number of animals in the vehicle versus the $\triangle 9$-THC groups $(\mathrm{n}=4){ }^{30}$

\section{Human studies}

To date, seven randomised controlled studies have examined the medicinal use of cannabis for reducing morbidity and mortality in people living with HIV. ${ }^{52}$ Interventions included smoked marijuana or hashish, ingested marijuana or hashish or ingested THC (dronabinol or other synthetic cannabinoid). Studies ranged from 21 to 84 days. Primary outcomes included HIV-related mortality (all cause) and morbidity (including type and duration of episodes of opportunistic infections, malignancies, incidence of AIDS and hospital admissions). Secondary outcomes included change in appetite, nausea, mood, pain, quality of life, body composition, haematological and nutritional markers, cognitive function, respiratory function and effect on pharmacokinetics of antiretrovirals and development of dependence or adverse sociological effects. The use of cannabis posed challenges for blinding due to the psychoactive effects. ${ }^{52}$

Only one study involving 62 patients examined the effects of cannabinoids on immune function and parameters associated with HIV infection. Participants in this study were assigned to either a $3.95 \%$ THC marijuana cigarette, a $2.5 \mathrm{mg}$ dronabinol capsule or a place capsule three times per day for 21 days. For the marijuana group, there was a statistically significant increase in CD4 counts from baseline versus placebo, and for the dronabinol group, there was a trend towards statistical significance. Neither CD4 nor CD8 cell counts were adversely affected, and the pharmacokinetic component of the study did not reveal any clinically significant interactions that would require dose adjustments of protease inhibitors (PIs). ${ }^{22}$ Adverse effects across studies included concentration difficulties, fatigue, sleepiness or sedation, increased duration of sleep, reduced salivation and thirst that improved on discontinuation. The authors of a systematic review on the use of cannabis for reducing morbidity and mortality in HIV concluded that: (1) evidence for substantial effects on morbidity and mortality is currently limited and (2) evidence for safety and efficacy of cannabis is lacking. Studies have been of short duration, in small numbers of patients and have focused on short-term measures of efficacy. ${ }^{52}$ Furthermore, no study examined the effects of cannabinoids on inflammatory markers or HIV reservoir markers through a randomised trial.

More recently, Manuzak et al published an observational study assessing the effect of cannabis use on peripheral immune cell frequency, activation and function in 198 people living with HIV. ${ }^{53}$ Individuals were grouped into heavy, medium or occasional cannabis users or non-cannabis users as determined by the quantify cannabis metabolite 11-nor-carboxy-tetrahydrocannabinol detected in plasma by mass spectrometry. They found that persons with heavy cannabis use had lower frequencies of HLA-DR+CD38+CD4+ and CD8+ T cell frequencies compared with people who did not consume cannabis. ${ }^{53}$ Furthermore, heavy cannabis use was associated with decreased frequencies of proinflammatory intermediate (CD14++CD16+) and non-classical (CD14+CD16+) monocyte subsets. ${ }^{53}$ They also documented a reduction in antigen-producing cells secreting proinflammatory cytokines IL-23 and tumour necrosis factor- $\alpha{ }^{53}$ Rizzo et al also demonstrated that levels of circulating CD16 monocytes and interferon-gamma-induced protein (IP)-10 from people living with HIV who either were or were not cannabis users. ${ }^{54}$ Lower levels of CD16+ monocytesand plasma IP-10 were found in cannabis users compared with non-cannabis users. ${ }^{54}$ However, this study did not quantify the level of cannabis exposure in the two groups. Although these studies demonstrated favourable associations between inflammation and cannabis use, it must be borne in mind that both of these studies were observational only. As these studies were not randomised controlled trials, it is possible that people living with HIV who used cannabis in these studies differed in other significant ways from PLWHIV who did not use cannabis.

\section{Study rationale}

Cannabis may hold many potential therapeutic benefits for people living with HIV due to its promising anti-inflammatory and antifibrotic effects. Before adequately powered interventional studies can be designed to study cannabis as a therapy for specific conditions associated with chronic inflammation and fibrosis, a key first step will be to demonstrate that cannabinoid use in a clinical trial is feasible and that they have a favourable safety and tolerability profile. As such, we propose a proof-of-concept pilot study to examine the feasibility, safety and tolerability of cannabinoid oils consumed orally in people living with HIV on effective ART. As a secondary objective, we will examine the effect of cannabinoid oils on immune profiles, including levels inflammatory markers associated with HIV disease progression and frequencies of activated and senescent $\mathrm{CD} 4$ and CD8 $\mathrm{T}$ cells. Frequencies of regulatory $\mathrm{T}$ cells and various subsets of Th17 will also be assessed. Finally, an exploratory objective will be to study the effect of cannabinoid oils on markers of HIV persistence and the composition of the gastrointestinal microbiome. 
We propose to use combination therapy of THC:CBD oils in capsule format (TN-TC11LM and TN-TC19LM capsules) ingested orally to examine these outcomes. Although research to date involving HIV/SIV has examined THC, data from in vitro, animal and human studies suggests that $\mathrm{CBD}$ has favourable anti-inflammatory effects, and the combination of CBD with THC increases tolerability. ${ }^{55-59}$

\section{METHODS/DESIGN}

\section{Study design}

This is a randomised, open-label, interventional study (the Canadian HIV Trials Network (CTN) number PT028) whereby capsules containing CBD:THC oils are consumed for 12 weeks to assess safety and tolerability. Their ability to reduce immune activation (as determined by percentage of activated CD8+CD38+HLA-DR+ T cells), size of the peripheral HIV reservoir and change in gastrointestinal microbiome composition will also be examined. Participants will continue to take their ART treatments as prescribed throughout the study.

\section{Setting}

Recruitment of participants will occur at the Chronic Viral Illness Service (CVIS), Royal Victoria Hospital (Glen campus) of the McGill University Health Centre (MUHC), the largest academic HIV clinic in Canada.

\section{Recruitment and enrolment}

Study staff at the CVIS will conduct chart reviews of prospective people living with HIV ahead of their clinic visits to determine which persons have had suppressed viral load for at least 3 years on ART. The patient chart will be flagged, and if the treating HIV physician believes the person to be suitable for the study, the physician or study staff will approach potential trial participants at their clinic visit. The trial staff will inform the patients about the trial and invite him or her for eligibility screening and possible trial enrolment. Participant eligibility will be documented and written informed consent obtained for eligible patients by the study coordinator. The study coordinator will systematically document all individuals who have been approached for the study in addition to reasons for acceptance and refusal to participate in the study. Individuals who wish to discuss their participation in the study with their treating physician and/or family and friends will have the opportunity to do and may enrol their next scheduled clinic visit. Following enrolment, participants will be followed during the study by the principal investigator and study coordinator at the CVIS.

\section{Inclusion criteria}

Eligible participants must meet the following criteria within 4 weeks prior to beginning the cannabinoid capsules to be considered eligible for study entry: (1) documented HIV infection by western blot, enzyme immunoassay or viral load assay; (2) aged 18 years or older; (3) viral load $<40$ copies $/ \mathrm{mL}$ for at least the last 3 years (maximum two blips $<500$ copies $/ \mathrm{mL}$ allowed); (4) no cannabinoid use for at least 1 month prior to enrolment with negative baseline cannabinoid urine screen; and (5) able to communicate in either English for French. We will attempt to enrol equal proportions of men who have sex with men (MSM) and heterosexual individuals.

\section{Exclusion criteria}

Individuals who meet any of the following criteria will be ineligible to participate: (1) using cannabinoid-containing products outside of the study or within 4 weeks of study commencement; (2) pregnant, breast feeding or planning to become pregnant during the course of the study; (3) enrolled in a separate study involving administration of medication, vitamin, supplement or herbal product; (4) active intravenous drug use; (5) active substance dependence; (6) prior history of hypersensitivity to cannabis or cannabis-containing products; (7) known or suspected allergy to sunflower lecithin oil; (8) active opportunistic infection or malignant condition; (9) unintentional weight loss of $10 \%$ or more of body weight in the last 6 months; (10) unstable angina or acute cardiac event in the past year; (11) active psychiatric disorder or history of psychiatric depression (other than mild depression or anxiety); (12) on antipsychotic medication; (13) known or suspected family history of schizophrenia or severe personality disorder; (14) serious cardiovascular disease such as ischaemic heart disease, arrhythmias, poorly controlled hypertension or severe heart failure; (15) anaemia (haemoglobin $<100 \mathrm{~g} / \mathrm{L}$ ); (16) active liver disease or unexplained persistent elevations of serum transaminases; (17) coinfection with hepatitis B or C (positive Hepatitis B Surface antigen (HBsAg) or positive anti-HBc (Hepatitis B Core) antibodies with a detectable Hepatitis B virus (HBV) DNA viral load or positive anti-HCV antibodies with a detectable Hepatitis C virus (HCV) RNA viral load); (18) alanine aminotransferase (ALT) or aspartate aminotransferase (AST) or alkaline phosphatase (ALP) $>2.5 \times$ upper limit of normal; (19) opportunistic infection in the last month as determined by the treating physician; (20) renal dysfunction; (21) unstable psychological or psychiatric condition as determined by the treating physician; (22) holding employment that requires operation of heavy machinery or which requires undergoing drug screening (ie, pilot or police officer); and (23) concurrent use within the past 8 weeks of anabolic hormones, prednisone, IL-2 or other agents known to alter immune function.

The investigators will review potential participants' current medication lists at the screening visit. If any concomitant therapy interacts with the study medication, and if this therapy cannot be substituted, that participant will not be eligible to enrol in the study. Although some HIV antiretroviral drugs/pharmacokinetic 'boosters' are metabolised by the CYP 1A2 and CYP3A4 (eg, ritonavir and cobicistat-boosted PIs), suggesting that a drug interaction may occur, in real practice at the CVIS, no clinically 


\begin{tabular}{|c|c|c|}
\hline Weeks & Daily dose & Number of capsules \\
\hline 1 & $\begin{array}{l}5 \mathrm{mg} \text { THC/ } \\
5 \mathrm{mg} \mathrm{CBD}\end{array}$ & $\begin{array}{l}\text { One capsule twice daily, taken } \\
\text { orally. }\end{array}$ \\
\hline 2 & $\begin{array}{l}10 \mathrm{mg} \text { THC/ } \\
10 \mathrm{mg} \text { CBD }\end{array}$ & $\begin{array}{l}\text { Two capsules twice daily, } \\
\text { taken orally } \\
\text { (four capsules per day). }\end{array}$ \\
\hline 3 & $\begin{array}{l}15 \mathrm{mg} \text { THC/ } \\
15 \mathrm{mg} \text { CBD }\end{array}$ & $\begin{array}{l}\text { Two capsule three times daily, } \\
\text { taken orally } \\
\text { (six capsules per day). }\end{array}$ \\
\hline 4 & $\begin{array}{l}20 \mathrm{mg} \text { THC/ } \\
20 \mathrm{mg} \text { CBD }\end{array}$ & $\begin{array}{l}\text { Two capsules four times daily, } \\
\text { taken orally } \\
\text { (eight capsules per day). }\end{array}$ \\
\hline $5-12$ & $\begin{array}{l}25 \mathrm{mg} \\
\mathrm{THC} / 25 \mathrm{mg} \mathrm{CBD}\end{array}$ & $\begin{array}{l}\text { Two capsules } 5 \text { times daily, } \\
\text { taken orally } \\
\text { (10 capsules per day). }\end{array}$ \\
\hline
\end{tabular}

Group 1: low CBD dose TN-TC11LM oral capsules $(2.5 \mathrm{mg}$ $\mathrm{THC} / 2.5 \mathrm{mg}$ CBD capsules). This group will be advised to start by taking one capsule twice daily for 1 week ( $5 \mathrm{mg}$ THC/5 mg CBD) and increase the number of capsules as tolerated to a maximum of 10 capsules daily by weeks $5-12$ ( $25 \mathrm{mg}$ THC/25 mg CBD total per day). Participants will record the times and dates of all capsules consumed in a logbook.

CBD, cannabidiol; THC, tetrahydrocannabinol.

significant drug interactions have been observed in patients reporting heavy cannabis use. Therefore, individuals will not be precluded to participate based on their specific antiretroviral regimen.

\section{Study intervention}

The study medications are TN-TC11LM and TN-TC19LM capsules that contain THC:CBD in a ratio of $1: 1$ $(2.5 \mathrm{mg} / 2.5 \mathrm{mg})$ and $1: 9(5 \mathrm{mg} / 45 \mathrm{mg})$, respectively. These study drugs are being provided by Tilray, and the active pharmaceutical ingredients are extracted from the cannabis plant and purified according to pharmaceutical standards $(>98 \%)$. Participants will be advised to gradually increase the number of capsules they take based on the suggested titration scheme presented in tables 1 and 2 , until a daily maximum is reached. These maximum amounts are composed of 10 capsules of TN-TC11LM (25 mg THC/25 mg CBD total per day) or 3 capsules of TN-TC19LM (15 mg THC: $135 \mathrm{mg}$ CBD for TN-CT19L) per day. These doses were selected as in a clinical trial for neuropathic pain; doses equivalent to $2.5 \mathrm{mg}$ of THC were well tolerated. ${ }^{34}$ More recently, among patients (ages 2-55 years) with the Lennox-Gastaut syndrome, CBD at a dose of 10 or $20 \mathrm{mg}$ per $\mathrm{kg}$ per day resulted in greater reductions in the frequency of drop seizures than placebo and was well tolerated overall other than for an increase liver aminotransferase concentrations ${ }^{60}$ Due to person-to-person variability in the ability to metabolise and tolerate cannabinoids, ${ }^{61}$ we have opted for patients to titrate their dose of medication to a range where they are comfortable as the titration method of dosing has proven successful in other clinical trials involving cannabinoids. ${ }^{34}$
Table 2 Suggested titration schedule for group 2: high CBD dose TN-TC19LM oral capsules

\begin{tabular}{|c|c|c|}
\hline Weeks & Daily dose & Number of capsules \\
\hline 1 & $\begin{array}{l}5 \mathrm{mg} \text { THC/ } \\
45 \mathrm{mg} \mathrm{CBD}\end{array}$ & One capsule daily, taken orally. \\
\hline 2 & $\begin{array}{l}10 \mathrm{mg} \text { THC/ } \\
90 \mathrm{mg} \text { CBD }\end{array}$ & $\begin{array}{l}\text { One capsule twice daily, taken } \\
\text { orally } \\
\text { (two capsules per day). }\end{array}$ \\
\hline 3 & $\begin{array}{l}15 \mathrm{mg} \text { THC/ } \\
135 \mathrm{mg} \mathrm{CBD}\end{array}$ & $\begin{array}{l}\text { One capsule three times daily, } \\
\text { taken orally } \\
\text { (three capsules daily). }\end{array}$ \\
\hline 4 & $\begin{array}{l}20 \mathrm{mg} \text { THC/ } \\
180 \mathrm{mg} \text { CBD }\end{array}$ & $\begin{array}{l}\text { One capsule four times daily, } \\
\text { taken orally } \\
\text { (four capsules). }\end{array}$ \\
\hline $5-12$ & $\begin{array}{l}25 \mathrm{mg} \text { THC/ } \\
225 \mathrm{mg} \text { CBD }\end{array}$ & $\begin{array}{l}\text { One capsule five times daily, } \\
\text { taken orally } \\
\text { (five capsules). }\end{array}$ \\
\hline
\end{tabular}

Group 2: high CBD dose TN-TC19LM (5 mg THC/45 mg CBD capsules). This group will be advised to start by taking one capsule once daily for 1 week ( $5 \mathrm{mg}$ THC/45 mg CBD) and increase the number of capsules as tolerated to a maximum of 10 capsules daily by week 5 ( $25 \mathrm{mg}$ THC/225 mg CBD total). Participants will record the times and dates of all capsules consumed in a log book. $\mathrm{CBD}$, cannabidiol; THC, tetrahydrocannabinol.

\section{Randomisation}

After eligibility is confirmed and written informed consent obtained, participants will be randomised to either TN-TC11LM (group 1) or TN-TC19LM (group 2) capsules, which contain THC:CBD in a ratio of $1: 1$ and $1: 9$, respectively. Prior to study commencement, a statistician unassociated with the study will develop a randomisation scheme using SAS and input into a password-protected web-based randomisation system. Variable block sizes of 2 and 4 will be used. Participants will be assigned to either group 1 versus group 2 based on the predesignated allocation code. As this is an unblinded study, participants and study staff will be aware of the group to which the participant has been randomised. A computerised audit trail will track date and time of allocation, patient study identification number and treatment allocation. The randomisation group will be recorded in the study log, which will be accessible to the sponsor/medical manager and study coordinator.

\section{Measurements}

At the screening visit, clinical information will be collected from each participant including age, ethnicity, sexual orientation, list of current medications, dosage, date of treatment initiation, any antimicrobials taken in the previous 6 months, psychiatric disorders, duration of HIV infection, current ART regimen and duration of ART regimen months, ART history, CD4+ T cells count within the past 3 months, nadir CD4+ T cells count, CD4/CD8 ratio, duration of plasma viral load suppression and any pre-existing medical conditions, signs or symptoms. Information will also be collected on whether the individual 
consumed cannabis in the past, the form(s) in which it was consumed, frequency of use and reasons for use.

\section{Clinical parameters}

Scheduled visits will occur to monitor safety and tolerability, as per the visit schedule depicted in table 3. Visits will include physical exam with vital signs, weight, occurrence of adverse events (AEs; and concomitant medications) and the presence of common symptoms associated with cannabinoids including dizziness, nausea, headaches, appetite or mood changes. At visits, blood for some or all of the following will be collected: CD4+ T cells count, CD8+ T cells, CD4/CD8 ratio, plasma viral load, complete blood count, AST, ALT, ALP, total bilirubin, urea, creatinine and blood glucose, $\mathrm{T}$ cell activation and inflammatory markers and testing for syphilis if the participant tested positive during the 4 weeks prior to beginning consuming the study capsules. A stool sample for analysis of the bacterial and fungal microbiome assessment will also be collected prior to beginning the study capsules. Participants will be enrolled in the study for up to 15 weeks but will consume capsules for a period of 12 weeks. Participants will undergo screening tests and eligibility assessment within 4 weeks prior to initiating study capsules. Participants will then undergo assessments after the first week of capsule consumption and every 2 weeks thereafter. A second stool sample for bacterial and fungal microbiome analysis will be collected during the final week of capsule consumption. The final visit will occur 2 weeks after study drug cessation.

\section{Medication adherence}

Participants will be asked to keep a log book in which they enter the number of TN-TC11LM or TN-TC19LM capsules consumed, the time, as well any AEs they noted and the timing of these AEs relative to capsule intake. Furthermore, individuals will be asked to record whether they took their ART that day or whether any doses were missed. Participants will be asked to bring their log books with them to study visits and the coordinator will photocopy this information.

\section{Quality of life and mood assessment}

Questionnaires measuring quality of life (WHO Quality of Life HIV-BEF (WHOQOLHIV-BREF) and the EuroQol-5Dimension (EQ-5D)) and mood (Profile of Mood States (POMS) will be administered at baseline, midway through the study and at the end of the study, as outlined in table 3. WHOQOLHIV-BREF consists of 31 items that measure the following domains: physical health, psychological health, social relationships and environment. It is a shorter version of the original instrument (WHOQOL) and is more convenient for use in clinical trials, taking approximately $10 \mathrm{~min}$ to complete. EQ-5D is a descriptive questionnaire examining five dimensions: (1) mobility, (2) self-care, (3) usual activities, (4) pain/ discomfort and (5) anxiety/depression. Each dimension has five levels: no problem, slight problems, moderate problems, severe problems and extreme problems. The participant indicates the state of his his or her health by ticking the box most appropriate to the statement in each of the five dimensions. This decision results in a one-digit number that expresses the level selected for that dimension. The digits for the five dimensions can be combined into a five-digit number that describes the participant's health state. It takes about $10 \mathrm{~min}$ to complete. The POMS questionnaire measures the following six factors: (1) tension-anxiety, (2) anger-hostility, (3) fatigue-inertia, (4) depression-dejection, (5) vigour-activity and (6) confusion-bewilderment. It is very sensitive to non-clinical changes in mood states and takes approximately 5 min to complete. These questionnaires will be administered by a trained research coordinator.

\section{Research hypothesis}

THC:CBD oils consumed orally-as TN-TC11LM and TN-TC19LM oral capsules-will be safe and well tolerated in PLWHIV. They will also be associated with a reduction in markers of inflammation, reduction in frequency of activated $\mathrm{T}$ cells and reduction in HIV reservoir size.

\section{Study outcome measures}

The primary objective is to evaluate the safety and tolerability of TN-TC11LM and TN-TC19LM oral capsules in PLWHIV on effective ART. The primary between group comparison is the percentage of participants without any signs of significant toxicity; percentage of participants who are able to complete the study and scores on the WHOQOLHIV-BREF Scale, EQ-D5 and POMS questionnaires from week 0 to week 12 are secondary outcomes that will also be compared between groups. The secondary objective is to determine the effect of TN-TC11LM and TN-TC19LM oral capsules on frequency of activated T-cells and markers of inflammation association with HIV disease progression. Exploratory objectives are to determine the effect of TN-TC11LM and TN-TC19LM oral capsules on: (1) the size of the peripheral HIV reservoir and (2) the composition of the gastrointestinal bacterial and fungal microbiome.

\section{Safety and tolerability}

Safety will be assessed at regular intervals (table 3) by vital signs and AEs monitoring, as reported by the participant and actively sought at each study visit by the coordinator or physician. Biological safety will be evaluated by haematology, biochemistry and other clinical, laboratory or other diagnostic tests done on participants during the course of the study. Lab results for all participants for assessed safety variables will be reviewed by the trial investigator. The Data Safety Monitor will also review safety information. Toxicity of TN-TC11LM and TN-TC19LM will be assessed using the WHO toxicity scale. All AEs, regardless of the grade, will be documented, and it will be noted whether these symptoms were already present at baseline. Any AEs that occur during the study will be evaluated by the trial investigators, and grades 3 and 4 


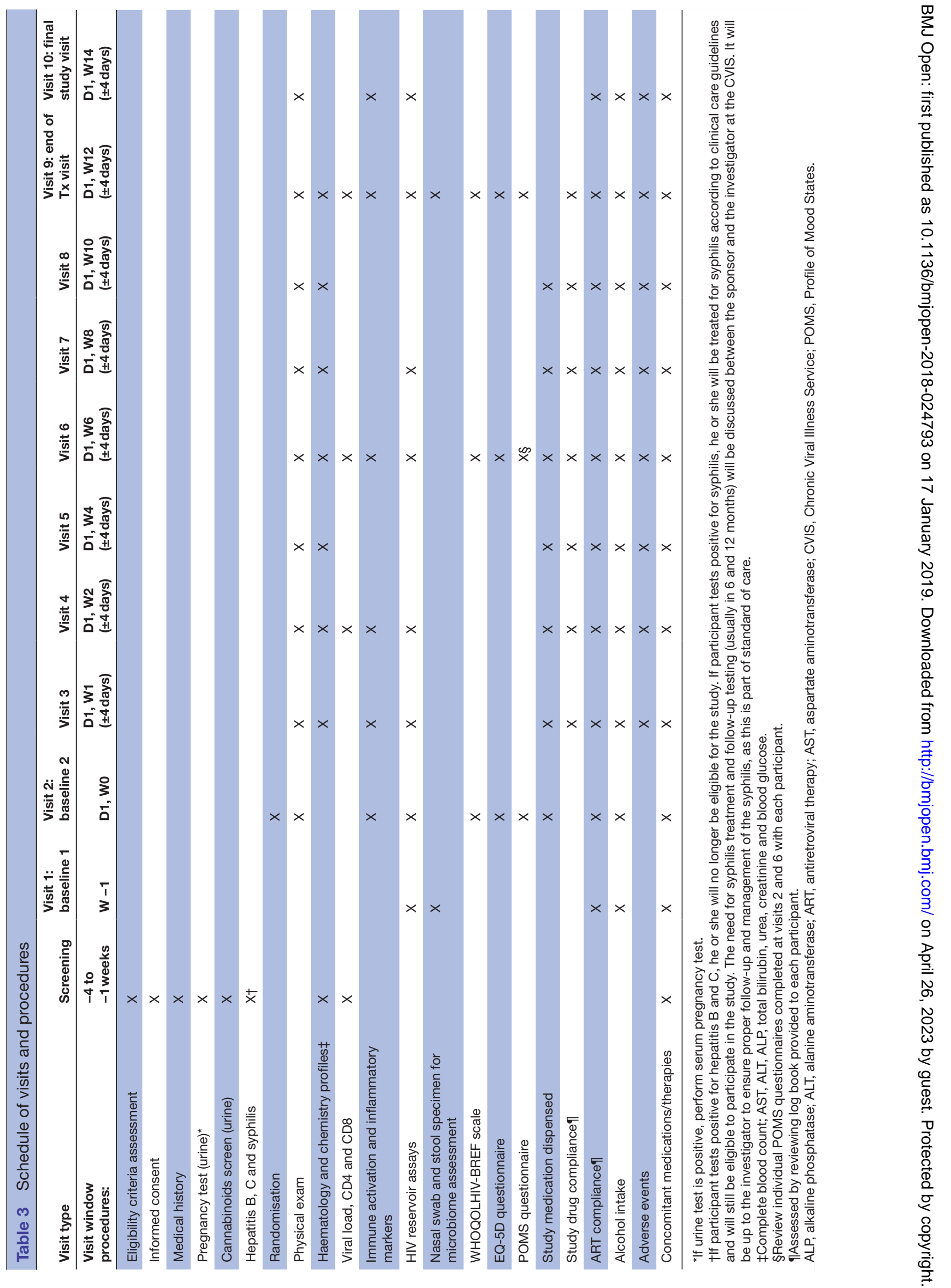


AEs will be recorded on the CRFs. If required, blood specimens will be collected for haematology and biochemistry tests. Participants having AEs will be monitored with relevant clinical assessments and laboratory tests, as determined by the trial investigator. The trial investigator will report ongoing AEs at the completion of the clinical study to the primary treating physician at the CVIS who will determine the need for and provide standard medical care. The trial investigator will ensure that the event is satisfactorily resolved or that no additional follow-up is needed. Any participant who discontinues the study for an unresolved clinically significant $\mathrm{AE}$ will be followed until satisfactory clinical resolution is achieved and the $\mathrm{AE}$ recorded on the case report form (CRF), regardless of severity grading. AEs that may be related to TN-TC11LM and TN-TC19LM will be managed by dose reduction of TN-TC11LM and TN-TC19LM. TN-TC11LM and TN-TC19LM will be discontinued permanently in the event of any life-threatening AEs.

\section{$T$ cell subsets and immune activation}

The frequency of different CD4+ and CD8+ T cell subsets will be defined using multiparameter flow cytometry (BD Fortessa X-20) in peripheral blood. The expressions of CD3, CD4 and/or CD8, CD45RA, CCR7 and CD27 will be used to measure the frequency of naive $\left(\mathrm{CD} 45 \mathrm{RA}^{+} \mathrm{C}\right.$ $\left.\mathrm{CR} 7^{+} \mathrm{CD} 27^{+}\right)$, central memory $\left(\mathrm{CD} 45 \mathrm{RA}^{-} \mathrm{CCR} 7^{+} \mathrm{CD} 27^{+}\right)$, transitional memory $\left(\mathrm{CD} 45 \mathrm{RA}^{-} \mathrm{CCR} 7^{-} \mathrm{CD} 27^{+}\right)$, effector memory (CD45RA ${ }^{-} \mathrm{CCR} 7^{-} \mathrm{CD}^{2} 7^{-}$) and terminally differentiated $\left(\mathrm{CD} 45 \mathrm{RA}^{+} \mathrm{CCR} 7^{-} \mathrm{CD} 27^{-}\right)$cells. Regulatory $\mathrm{T}$ (Treg) cells will be defined as $\mathrm{CD} 3^{+} \mathrm{CD} 4^{+} \mathrm{CD} 25^{\text {high }} \mathrm{FoxP} 3{ }^{\text {high }} \mathrm{C}$ D127 ${ }^{\text {low }}$ cells. Expression of the CD39 and CD73 ectoenzymes involved in Treg-mediated immunosuppression and HIV disease progression (via the adenosine pathway) will be also assessed. ${ }^{62}{ }^{63}$ Various subsets of Th cells (T helper) will be defined as Th1 (CD45RA $\left.{ }^{-} \mathrm{CCR} 6^{-} \mathrm{CCR} 4^{-} \mathrm{CXCR}^{+}\right)$, Th2 (CD45RA $\left.{ }^{-} \mathrm{CCR}^{-} \mathrm{CCR}^{+}{ }^{+} \mathrm{CXCR}^{-}\right)$, Th17 (CD45RA ${ }^{-} \mathrm{C}-$ $\left.\mathrm{CR}^{+} \mathrm{CCR} 4^{+} \mathrm{CXCR}^{-}\right)$and Th1/Th17 (CD45RA ${ }^{-} \mathrm{CCR} 6^{+} \mathrm{C}^{-}$ $\mathrm{CR}^{-} \mathrm{CXCR}^{+}$). Levels of CD8+ and CD4+ Tcell immune activation (CD38/HLA-DR coexpression) and senescence $\left(\mathrm{CD} 28^{-} \mathrm{CD} 57^{+}\right)$will be also assessed on all $\mathrm{T}$ cell subsets. These markers will be assessed at week $0,1,2,6$, 12 and 14.

\section{Inflammatory markers assessments}

Plasma levels of various inflammatory markers including interferon- $\alpha$, IL-1 $\beta$, IL-6, IL-10, IL-17, TGF- $\beta$ and IP-10 will be assessed via Luminex (Millipore) and levels of d-dimer, $\mathrm{C}$ reactive protein and markers of microbial translocation LPS and sCD14 will be assessed by ELISA in batch from blood drawn at weeks 0, 1, 2, 6, 12 and 14 .

\section{Peripheral blood HIV reservoir size}

Blood for HIV reservoir assessment will be collected at two time points prior to cannabis initiation (1 week prior to cannabinoid capsule initiation and immediately prior to cannabinoid capsule initiation) to account for normal fluctuations in baseline levels of HIV persistence markers.
Subsequently, virological measures will be done at weeks $1,2,6,8,12$ and 14. CD4+ T cells isolated form peripheral blood mononuclear cells (PBMCs) by magnetic negative selection. To capture all infected cells, the frequency of cells harbouring total and integrated HIV DNA will be measured using well-established assays on a total of 500000 cells (sensitivity of 1 copy/reaction). ${ }^{1764}$ As most of the HIV genomes are defective, the recently developed 'tat/rev induced limiting dilution assay' (TILDA), which provides a more functional measurement of the HIV reservoir, ${ }^{65}$ will be employed. To assess if residual levels of viral replication may occur, we will measure 2-LTR circles, which are proposed to be a surrogate marker for ongoing HIV replication during ART. ${ }^{64667}$ Specifically, this combination of assays will indicate if cannabis has an impact on the size of the total reservoir (DNA), the functional reservoir (TILDA) and ongoing viral replication (2-LTR circles). Measurements will be performed in batch.

\section{Gastrointestinal microbiome composition}

A stool sample without preservative will be collected from each participant at the beginning of the study prior to consuming the capsules and during the final week (week 12) of capsule consumption. Specimens will be stored at $-80^{\circ} \mathrm{C}$ until analysed in batch, as previously described. ${ }^{68}$ Bacterial DNA will be extracted with PCR amplified targeting of the 16S rRNA gene using universal primers that flank the V3-V4 region of the $16 \mathrm{~S}$ gene modified with the addition of TruSeq Illumina adapters, also as previously outlined. ${ }^{69}$ Internal transcribed spacer for fungal DNA extraction will be used for fungi. PCR amplification, PCR amplicon quantification and sequencing will be performed as previously described. ${ }^{68} 69$

\section{Sample size and statistical analyses}

In this proof-of-concept study, we are exploring a phenomenon with little in vivo data and with a limited study budget. For this reason, we have chosen a convenience sample of 26 participants, 13 per arm, without formal power calculations. This number of individuals will enable us to assess feasibility (willingness of patients to participate, attend study visits and complete questionnaires and numbers of drop-out participants) as well as safety and tolerability. The data obtained will help to guide future sample size calculations for future studies. Although the small number of participants may result in wide CIs for AEs, this number of participants should give us an idea about the degree of variability for continuous outcomes we are measuring.

For the primary endpoint, the proportions of participants without any signs of significant toxicity (grades 0-2 scores on the WHO toxicity scale), proportions of participants who complete the study and scores on the WHOQOLHIV-BREF, EQ-5D and POMS questionnaires will be examined using descriptive statistics. We will also compare these proportions for group 1 versus group 2 using a Fisher's exact test. For quality of life and mood measures, we will use analysis of covariance with 12-week 
score as outcome and baseline score as covariate and treatment as independent variable. With regards to the POMS questionnaire, we will consider only overall scores (and not subscores) due to the small sample size that would make comparisons of the subscale inappropriate.

Immune activation levels for groups 1 versus group 2 at week 12 will be compared using analysis of covariance with adjustment for the week 0 activation levels. The mean change and associated 95\% CI will be reported for each of the secondary endpoints described above. Furthermore, the study drug treatment period will be compared with the baseline period with each arm. If the treatment effect is similar within the two arms, then an analysis of the treatment effect will be pooled over the two arms, using analysis of covariance. The change is immune activation levels following discontinuation of study drug (ie, from week 12 to week 14) will be reported as a mean with corresponding 95\% CI. A 50\% reduction will be considered significant. ${ }^{70}$

Analogous analyses will be conduction for reservoir assessments other outcome measures listed above as endpoints. A signed rank (non-parametric) test will be used to compare number of copies of total and integrated DNA at baseline 2 versus at week 12. Group differences in the change of HIV reservoir size from baseline to 12 weeks will be assessed by the Mann-Whitney $\mathrm{U}$ test. Wilcoxon signed-rank test will be used to compare the HIV reservoir and inflammatory markers in blood samples of the same patient from baseline to 12 weeks. At least a twofold decrease in frequency of infected cells in both groups from baseline to 12 weeks of treatment will be considered significant. ${ }^{71}$ Microbiome composition will be described with regards to the frequencies of microorganisms families for the groups at baseline and then at 12 weeks of treatment. Due to the exploratory nature of this objective, no formal statistics will be applied.

\section{Patient and public involvement}

The CTN Community Advisory Committee (CAC) was involved in the peer-review process of this study proposal, deemed that the research questions addressed were of very high priority to people living with HIV and voted for the funding of this study. The CAC's critiques of the initial proposal were taken into account in the revised proposal. Two members of the CAC (SM and EM) were involved in finalising the study design, inclusion/exclusion criteria, outcome measures and monitoring plans and are formal study investigators and coauthors. Preliminary and final results of the study will be shared with community members, patient participant and the public at biannual CTN meetings, through the CTN newsletter and on the CTN website in addition to the annual Canadian Association of HIV Research meeting.

\section{Data management}

All clinical data and electronic files will be stored in the secure environment of the CVIS of the MUHC. All data published will be anonymised. Only researchers affiliated with the study will have access to participant data. Study progress and safety will be evaluated in an ongoing fashion by the principal investigators and coinvestigators. The study will be monitored for safety and ongoing progress by a standing Data and Safety Monitoring Committee of clinicians and methodologists established by the Canadian HIV Trials Network. The committee meets every 6 months or as needed.

\section{Storage of biological specimens}

Within 1 hour of being drawn, blood from the CVIS will be transferred to the laboratory at the Research Institute of the McGill University Health Centre (RI-MUHC) (in the adjacent, connected building), and the plasma will be separated from PBMCs by Ficoll density centrifugation by an experienced laboratory personnel. PBMCs and plasma will be stored in liquid nitrogen tanks at the RI-MUHC laboratory until time for analysis. Patients will be contacted the day before their clinic visit to remind them when a stool specimen is due. They will be instructed to record the time of the provision of the sample on the paper bag containing the sterile container and store it in a refrigerator $\left(-4^{\circ} \mathrm{C}\right)$ until brought to the clinic. Once at the CVIS, the stool specimen will be placed in a large fridge designated specifically for the storage of stool specimens.

\section{Ethics and dissemination}

Written informed consent will be obtained from all study participants. The study protocol and informed consent are in the process of being reviewed by Health Canada's Therapeutic Product Directorate. The study will be conducted in accordance with the application Health Canada regulations, International Conference on Harmonization guidelines on current Good Clinical Practice and the Declaration of Helsinki. Patient enrolment for this trial is anticipated to begin August 2018.

Regardless of outcome, trial results will be disseminated through scientific peer-review publication, international and national conferences and the CTN according the Standard Protocol Items: Recommendations for Interventional Trials and Consolidated Standards of Reporting Trials guidelines for transparent reporting of trials. ${ }^{72} 73$ CTC will be responsible for initially drafting the manuscripts, and professional writers will not be used for any of the publications. Authorship will be determined based on criteria defined by the International Journal of Medical Editors. ${ }^{74}$ We aim to write the manuscript of the final results within 6 months of completing the study. Participants who have been involved in the trial will be given the option of having a summary of results sent to them.

\section{DISCUSSION}

Since the advent of ART, people living with HIV now have a longevity that approaches that of their HIV-uninfected counterparts but have a higher burden of non-communicable comorbidities including cardiovascular, pulmonary, renal and hepatic diseases. ${ }^{12}$ Heightened inflammation in 
people living with HIV despite ART is believed to be the driving before behind the increased rates of non-infectious comorbidities. Similarly, chronic immune activation fosters HIV persistence. ${ }^{10-15}$ As cannabinoids possesses both anti-inflammatory and antifibrotic properties, ${ }^{19}$ cannabinoids may represent a feasible method to reduce immune activation and enhance immune profile. This, in turn, may hasten the progression of non-opportunistic complications associated with HIV. Although some studies have examined whether there are beneficial effects on inflammation resulting from treatment with integrase inhibitors compared with PIs, between PIs and non-nucleoside reverse transcriptase inihibitors, between specific nucleoside reverse transcriptase inhibitors, or with maraviroc in ART-naive patients, to date insufficient to conclude that any class of antiretrovirals is superior to other classes of antiretrovirals with regards to effects on inflammation. ${ }^{75}$ Furthermore, cannabis may induce cytochrome P450 (CYP) 1A2 via activation of the aromatic hydrocarbon receptor. ${ }^{76}$ CYP3A4 inducers and inhibitors alter the pharmacokinetics of $\triangle 9$-THC and CBD when administered as $\triangle 9-\mathrm{THC} / \mathrm{CBD}$ oromucosal spray. To date only one study has ever examined the effects of cannabinoids on the pharmacokinetics of antiretrovirals. Kosel et $a l$ studied the pharmacokinetics of smoked marijuana and dronabinol in people living with HIV receiving either indinavir and nelfinavir (two PIs no longer used due to their toxicity and adverse effect profiles). ${ }^{77}$ Individuals on stable regimens of indinavir $800 \mathrm{mg}$ every 8 hours or nelfinavir $750 \mathrm{mg}$ three times daily were randomised to one of three treatment arms: (1) 3.95\% THC marijuana cigarettes; (2) dronabinol $2.5 \mathrm{mg}$ capsules; or (3) placebo capsules given three times daily. Although there were statistically significant decreases in maximum concentration (Cmax) of indinavir in the smoked marijuana arm, the size of the changes in the pharmacokinetic parameters of both indivnavir and nelfinavir was sufficiently small not to impose any shortterm clinical consequence. ${ }^{77}$ Furthermore, the investigators concluded that use of marijuana or dronabinol is unlikely to impact ART. ${ }^{77}$ For these reasons and based on clinical experience at our clinic, we have not precluded individuals on any particular antiretroviral regimens from participating in this study.

In this pilot study, our primary objective is to assess the safety and tolerability of TN-TC11LM and TN-TC91LM taken by people living with HIV on suppressive ART. We hypothesise that these agents will be safe and well tolerated in people living with HIV, given that similar products are safe and well-= tolerated in other populations. Sativex is currently licenced as an adjunctive treatment for symptomatic relived of spasticity in adult patients with multiple sclerosis (MS) who have not responded adequately to other therapy. ${ }^{78-80}$ It is an oromucosal spray containing CBD and THC in 1:1 ratio. Marinol, which is a synthetic THC-containing capsule, is currently used for the treatment of anorexia associated with weight loss in persons with AIDS and nausea and vomiting associated with cancer chemotherapy in patients with insufficient response to conventional antiemetics. ${ }^{81}$ It has been estimated that 5472 patients have been exposed to Sativex, and there have been no safety concerns identified and the product remains well tolerated. The primary safety concerns of both Sativex and Marinol are consistent with the known pharmacological activity of cannabinoids. The primary safety concerns associated with Sativex included abuse potential, cardiovascular effects and central nervous system AEs. ${ }^{82}$ Although there is some evidence to suggest that individuals can develop 'cannabis use disorder', individuals do not develop the same extremes of behaviour as observed with other drugs of abuse. ${ }^{83}$ In two clinical trials, nabiximols such as Sativex have been used in two clinical trials whereby treatment was abruptly ceased to study whether withdrawal symptoms would develop ${ }^{84}$ In both studies, no withdrawal syndromes were observed. ${ }^{86}$ Cannabinoids have cardiovascular effects that include tachycardia and fluctuations in blood pressure, including episodes of postural hypotension. Therefore, these agents should not be used in patients with serious cardiovascular disease, such as ischaemic heart disease, arrhythmias, poorly controlled hypertension or severe heart failure. THC has complex effects on the CNS and should not be used in patients with a personal or strong family history of psychosis. Examples of such conditions include schizophrenia and affective psychosis since symptoms of these disorders may be aggravated by cannabinoids. In Patients with MS receiving Sativex in clinical studies, psychiatric-related adverse effects included disorientation, depression including depressed mood, dissociation, euphoric mood, hallucination, hallucinations (auditory and visual), illusions, paranoia, suicidal ideation and delusional perception. ${ }^{87}$ Interestingly, there is some evidence to demonstrate that CBD may actually improve psychotic symptoms in persons suffering from schizophrenia. $^{88}$

For Marinol, which contains only THC, the most frequency reported adverse effects experienced by patients with AIDS during placebo controlled clinical trials involved CNS and were reported in $33 \%$ of patients receiving Marinol. About 25\% reported a CNS AE during the first 2 weeks and about $4 \%$ reported such an event each week for the next 6 weeks thereafter. ${ }^{89}$ By combining CBD with THC, we anticipate that tolerability will be greatly enhanced. When combined with THC, CBD reduces the risk for many adverse effects. ${ }^{88}$ Furthermore, individuals will be instructed to titrate up the dose based on their own tolerability and reduce the dose if they experience any undesirable effects. Furthermore, due to the extremely low levels of CB1 receptors in the brainstem, ${ }^{90}$ death due to overdosing on cannabis or cannabinoids alone has never been described.

The study medications and doses were chosen after a lengthy review of the existing literature and discussion with experts in the field of pain management. A high degree of interindividual variability in metabolism following administration of cannabinoids is observed due 
to polymorphisms in cytochrome isoenzymes. ${ }^{61}$ Given that the therapeutic doses of cannabinoids are highly variable between individuals, a dose titration schedules are usually recommended. When used to treat specific conditions, persons may be told to increase the dose until they achieve adequate symptom relief without adverse effects. This method was observed to work well when used in the first cohort study on the long-term safety of medicinal cannabis for non-cancer chronic pain in seven Canadian clinics. ${ }^{34}$

Given this is a pilot study and given our budgetary restrictions, a convenience sample of 26 participants was selected without formal power calculation. If this study demonstrated that TN-TC11LM and/or TN-TC91LM are safe and tolerable in people living with HIV and can reduce systemic inflammation, future studies will be performed to address the potential of these agents to ameliorate specific conditions in people living with HIV. Should future studies be conducted, data generated from this trial will assist with power calculations. Similar to a study conducted by members of our group on the ability of Niaspan (extended-release niacin) to reduce immune activation, as determined by percentage of activated CD8+ CD38+ HLA-DR+ Tcells, we decided that a $50 \%$ reduction in activated CD8+ CD38+ HLA-DR+ T cells would be considered significant. ${ }^{70}$ This is based on previous reports indicating that a 10 -fold difference exists between uninfected healthy controls and treated aviraemic HIV-infected individuals in level of activated CD8 ${ }^{+}$CD 38+ HLA-DR+ T cells. ${ }^{91}{ }^{92}$ All of the inflammatory mediators we selected for this study are known to drive immune activation. ${ }^{1693}$

In addition, we decided to make our objective examining the ability of TN-TC11LM and TN-TC91LM capsules to reduce the HIV reservoir, through the reduction of systemic inflammation, an exploratory objective. It is unclear if 3 months of treatment will be long enough to produce any meaningful reduction in the size of the HIV reservoir. Furthermore, it is unclear what reduction in reservoir size is required to have a meaningful effect on clinical outcomes. As mentioned earlier, we will consider a $50 \%$ decrease in the number of HIV-infected cells at baseline versus week 12 to be a significant reduction in the reservoir based on a study by Hill et al. ${ }^{71}$ To our knowledge, there is no other randomised clinical trial examining the effect of cannabinoids on inflammation and HIV reservoir size in people living with HIV.

Our study is unique in being the first randomised trial in the world to examine the association between ingestion of precise quantities of cannabinoids and effect on inflammation and peripheral HIV reservoir size. It is also noteworthy that we chose not to have a placebo arm as the effects of psychoactive effects of THC would be difficult to camouflage. Furthermore, we chose to use oral formulations of cannabinoids so that we could precisely control the dose ingested by participants. The active pharmaceutical ingredients are extracted from the cannabis plant and purified according to pharmaceutical standards $(>98 \%)$. This level of purity will enable investigators to know that the dosing provided is accurate and quantifiable and will also enable us to draw conclusions about the efficacy of the active ingredients being studying. When cannabis is smoked or vaped, there is variability in the methods and duration of inhalation used by participants that can influence dosage of cannabis ingested. The oral administration option also removes undesirable pulmonary effects such as symptoms wheezing or breathlessness in addition to inhalation of toxic chemicals. ${ }^{94}$ Of special interest to our group is the recent discovery that administration of oral cannabis with lipids leads to high levels of cannabinoids in the intestinal lymphatic system and prominent immunomodulation, as demontrated by Zgair $e t a l .^{20}$ This finding is especially important given the prominent role of the mesenteric lymph nodes and gut to HIV persistence. ${ }^{7}$ If oral cannabinoids can modify gut microbiome and the enteric immune system favourably, larger clinical trials could be conducted to examine this phenomena in further detail. As gut microbiota differs by sexual orientation, we are attempting to enrol approximately equal numbers of MSM as well as heterosexual individuals ${ }^{95}$ and will describe the demographics of the individuals enrolled in our study in detail. Similarly, we will report participants' current and nadir CD4 T counts as, in some studies, the enteric bacterial microbiome of patients with lower CD4 T counts exhibited reduced phylogenetic diversity and richness. ${ }^{96}$ There were increases in Enterobacteriaceae, which have been associated with inflammation. Therefore, immunodeficiency in progressive HIV infection is associated with alterations in the enteric virome and bacterial microbiome. ${ }^{96}$

The Canadian government has declared that cannabis' regulatory status will change from being an illegal substance to that of a legal substance in October 2018. Cannabis' change in regulatory status will likely stimulate more discussion among patients and physicians and thus physicians need to be informed about the potential risks and benefits of cannabis use. The change in the regulatory landscape will likely also foster more research into cannabis' therapeutic potential. We hope that this study will be a stimulus towards more open discussion between patients and their physicians and that it will reduce stigma associated with cannabinoids use. We also hope that this study will be the cornerstone for future studies investigating the therapeutic benefits of cannabis in PLWHIV and its potential at the individual level and at the population level in the form of harm reduction strategies.

\section{Author affiliations}

${ }^{1}$ Chronic Viral IIIness Service, McGill University Health Centre, Montreal, Canada ${ }^{2}$ Research Institute of McGill University Health Centre, Montreal, Canada

${ }^{3}$ Canadian Institutes of Health Research Canadian HIV Trials Network, Vancouver, British Columbia, Canada

${ }^{4}$ WILLL Cannabis Group, Toronto, Canada

${ }^{5}$ School of Population and Public Health, University of British Columbia, Vancouver, British Columbia, Canada 
${ }^{6}$ Department of Family Medicine, McGill University Health Centre, Montreal, Canada ${ }^{7}$ Department of Psychiatry, McGill University Health Centre, Montreal, Canada ${ }^{8}$ Centre de Recherche du CHUM and Department of Microbiology, Infectiology and Immunology, Université de Montréal, Montreal, Canada

${ }^{9}$ Department of Biological Sciences and BioMed Research Centre, University of Quebec at Montreal (UQAM), Montreal, Quebec, Canada

Acknowledgements This clinical trial was reviewed by both the CTN Scientific Advisory Committee and the Community Advisory Committee and is supported by a competitive grant (CTN PT028). We also wish to acknowledge Ms Dana Nohyek, Ms Judy Needham, Ms Jacqueline Sas and the Community Advisory Committee of the CTN for ongoing support. In addition, we would like to thank Ms Hansi Peiris and Jonathan Roger of the CVIS for logistical and administrative assistance. Furthermore, we wish to acknowledge Tilray and notably Philippe Lucas and Catherine Jacobson for interest and support of this study through the provision of cannabinoid capsules and for assistance with the application to Health Canada.

Contributors CTC conceived and designed the study, drafted the grant and the protocol manuscript, will organise and supervise trial implementation and will be responsible for trial management. She will also be responsible for trial managements, staff training and supervision. M-AJ contributed to study design and participated in grant writing. ZS, BL, J-PR, JC, JaS, M-JB and MBK will participate in study implementation. M-JB provided input on questionnaires, while CTC and M-AJ provided immunological expertise and NC provided expertise related to the HIV reservoir. SM and EM contributed to study design. CTC, M-AJ and NC designed the experiments. JoS contributed to the statistical analysis plan. CTC, $\mathrm{M}-\mathrm{AJ}$ and NC designed the experiments. All authors participated in refinement of the study methods, critically reviewed the manuscript drafts and approved the final manuscript. The CTN provides regulatory support. CTC and BL are Fonds de recherche du Québec-Santé (FRQ-S) chercheur-boursier-clinicien junior 1. BL holds a Strategy for Patient-Oriented Research (SPOR) Mentorship Chair in Innovative Clinical Trials. J-PR holds the Louis Lowenstein Chair in Hematology and Oncology at McGill University. M-AJ is holder of a Tier 2 Canada Research Chair in immunovirology.

Funding This work is supported by the CIHR Canadian HIV Trials Network.

Competing interests Tilray Inc is supplying the study medications free of charge. All elements of the study are being undertaken independently of Tilray Inc. The authors declare there are no conflicts of interests.

Patient consent for publication Not required.

Ethics approval The study protocol and informed consent have been approved by the Research Ethics Board of the McGill University Health Centre (MUHC-2018-4336).

Provenance and peer review Not commissioned; externally peer reviewed.

Open access This is an open access article distributed in accordance with the Creative Commons Attribution Non Commercial (CC BY-NC 4.0) license, which permits others to distribute, remix, adapt, build upon this work non-commercially, and license their derivative works on different terms, provided the original work is properly cited, appropriate credit is given, any changes made indicated, and the use is non-commercial. See: http://creativecommons.org/licenses/by-nc/4.0/.

\section{REFERENCES}

1. Kalayjian RC, Machekano RN, Rizk N, et al. Pretreatment levels of soluble cellular receptors and interleukin- 6 are associated with HIV disease progression in subjects treated with highly active antiretroviral therapy. J Infect Dis 2010;201:1796-805.

2. Funderburg NT, Mayne E, Sieg SF, et al. Increased tissue factor expression on circulating monocytes in chronic HIV infection: relationship to in vivo coagulation and immune activation. Blood 2010;115:161-7.

3. The definition of emphysema. The definition of emphysema. Report of a National Heart, Lung, and Blood Institute, Division of Lung Diseases workshop. Am Rev Respir Dis 1985;132:182-5.

4. Brenchley JM, Price DA, Schacker TW, et al. Microbial translocation is a cause of systemic immune activation in chronic HIV infection. Nat Med 2006;12:1365-71.

5. Estes JD, Harris LD, Klatt NR, et al. Damaged intestinal epithelial integrity linked to microbial translocation in pathogenic simian immunodeficiency virus infections. PLoS Pathog 2010;6:e1001052.

6. Deeks SG, Tracy R, Douek DC. Systemic effects of inflammation on health during chronic HIV infection. Immunity 2013;39:633-45.
7. Costiniuk CT, Angel JB. Human immunodeficiency virus and the gastrointestinal immune system: does highly active antiretroviral therapy restore gut immunity? Mucosal Immunol 2012;5:596-604.

8. Dillon SM, Lee EJ, Donovan AM, et al. Enhancement of HIV1 infection and intestinal CD4+ T cell depletion ex vivo by gut microbes altered during chronic HIV-1 infection. Retrovirology 2016;13:5.

9. Vujkovic-Cvijin I, Dunham RM, Iwai S, et al. Dysbiosis of the gut microbiota is associated with HIV disease progression and tryptophan catabolism. Sci Trans/ Med 2013;5:ra91:193ra91.

10. El-Sadr WM, Lundgren J, Neaton JD, et al. CD4+ countguided interruption of antiretroviral treatment. $N$ Engl $J$ Med 2006;355:2283-96.

11. Baker JV, Peng G, Rapkin J, et al. Poor initial CD4+ recovery with antiretroviral therapy prolongs immune depletion and increases risk for AIDS and non-AIDS diseases. J Acquir Immune Defic Syndr 2008;48:541-6.

12. Baker JV, Peng G, Rapkin J, et al. CD4+ count and risk of nonAIDS diseases following initial treatment for HIV infection. AIDS 2008;22:841-8.

13. Kim H, Perelson AS. Viral and latent reservoir persistence in HIV-1infected patients on therapy. PLoS Comput Biol 2006;2:e135.

14. Lu W, Mehraj V, Vyboh K, et al. CD4:CD8 ratio as a frontier marker for clinical outcome, immune dysfunction and viral reservoir size in virologically suppressed HIV-positive patients. J Int AIDS Soc 2015;18:20052.

15. Rouzioux C, Richman D. How to best measure HIV reservoirs? Curr Opin HIV AIDS 2013;8:170-5.

16. Chun TW, Carruth L, Finzi D, et al. Quantification of latent tissue reservoirs and total body viral load in HIV-1 infection. Nature 1997;387:183-8.

17. Chomont N, El-Far M, Ancuta P, et al. HIV reservoir size and persistence are driven by $T$ cell survival and homeostatic proliferation. Nat Med 2009;15:893-900.

18. Chomont N, DaFonseca S, Vandergeeten $\mathrm{C}$, et al. Maintenance of CD4+ T-cell memory and HIV persistence: keeping memory, keeping HIV. Curr Opin HIV AIDS 2011;6:30-6.

19. Zurier RB, Cannabinoids BSH. inflammation, and fibrosis. FASEB journal: official publication of the Federation of American Societies for Experimental Biology 2016 (published Online First: 21 Jul 2016).

20. Zgair A, Lee JB, Wong JCM, et al. Oral administration of cannabis with lipids leads to high levels of cannabinoids in the intestinal lymphatic system and prominent immunomodulation. Sci Rep 2017;7:14542.

21. Costiniuk CT, Brunet L, Rollet-Kurhajec KC, et al. Tobacco smoking is not associated with accelerated liver disease in human immunodeficiency virus-hepatitis c coinfection: A longitudinal cohort analysis. Open Forum Infect Dis 2016;3:ofw050.

22. Abrams DI, Hilton JF, Leiser RJ, et al. Short-term effects of cannabinoids in patients with HIV-1 infection: a randomized, placebo-controlled clinical trial. Ann Intern Med 2003;139:258-66.

23. Lee $\mathrm{MH}$, Hancox RJ. Effects of smoking cannabis on lung function. Expert Rev Respir Med 2011;5:537-47.

24. Tahamtan A, Tavakoli-Yaraki M, Rygiel TP, et al. Effects of cannabinoids and their receptors on viral infections. J Med Virol 2016;88:1-12.

25. Smith TH, Sim-Selley LJ, Selley DE. Cannabinoid CB1 receptorinteracting proteins: novel targets for central nervous system drug discovery? Br J Pharmacol 2010;160:454-66.

26. DiPatrizio NV. Endocannabinoids in the Gut. Cannabis Cannabinoid Res 2016;1:67-77.

27. Muccioli GG, Naslain D, Bäckhed F, et al. The endocannabinoid system links gut microbiota to adipogenesis. Mol Syst Biol 2010;6:392.

28. Rietschel ET, Kirikae T, Schade FU, et al. Bacterial endotoxin: molecular relationships of structure to activity and function. Faseb $J$ 1994:8:217-25

29. Yao B, Mackie K. Endocannabinoid receptor pharmacology. Curr Top Behav Neurosci 2009;1:37-63.

30. Eisenstein TK, Meissler JJ. Effects of Cannabinoids on T-cell Function and Resistance to Infection. J Neuroimmune Pharmacol 2015;10:204-16.

31. Hanus L, Breuer A, Tchilibon S, et al. HU-308: a specific agonist for CB(2), a peripheral cannabinoid receptor. Proc Natl Acad Sci U S A 1999:96:14228-33

32. Russo EB. Cannabidiol Claims and Misconceptions. Trends Pharmacol Sci 2017;38:198-201.

33. Laprairie RB, Bagher AM, Kelly ME, et al. Cannabidiol is a negative allosteric modulator of the cannabinoid $\mathrm{CB} 1$ receptor. $\mathrm{Br} \mathrm{J}$ Pharmacol 2015;172:4790-805. 
34. Ware MA, Wang T, Shapiro S, et al. Cannabis for the Management of Pain: Assessment of Safety Study (COMPASS). J Pain 2015;16:1233-42.

35. Abrams DI, Jay CA, Shade SB, et al. Cannabis in painful HIVassociated sensory neuropathy: a randomized placebo-controlled trial. Neurology 2007;68:515-21.

36. Wilsey B, Marcotte T, Tsodikov A, et al. A randomized, placebocontrolled, crossover trial of cannabis cigarettes in neuropathic pain. $J$ Pain 2008;9:506-21.

37. Steffens S, Veillard NR, Arnaud C, et al. Low dose oral cannabinoid therapy reduces progression of atherosclerosis in mice. Nature 2005:434:782-6.

38. Franz CA, Frishman WH. Marijuana use and cardiovascular disease. Cardiol Rev 2016;24:158-62.

39. Mallat A, Lotersztajn S. Endocannabinoids and liver disease. I. Endocannabinoids and their receptors in the liver. Am J Physiol Gastrointest Liver Physiol 2008;294:G9-G12.

40. Mallat A, Teixeira-Clerc F, Deveaux V, et al. The endocannabinoid system as a key mediator during liver diseases: new insights and therapeutic openings. Br J Pharmacol 2011;163:1432-40.

41. Teixeira-Clerc F, Belot M-P, Manin S, et al. Beneficial paracrine effects of cannabinoid receptor 2 on liver injury and regeneration. Hepatology 2010;52:1046-59.

42. Muñoz-Luque J, Ros J, Fernández-Varo G, et al. Regression of fibrosis after chronic stimulation of cannabinoid $\mathrm{CB} 2$ receptor in cirrhotic rats. J Pharmacol Exp Ther 2008;324:475-83.

43. Tam J, Vemuri VK, Liu J, et al. Peripheral CB1 cannabinoid receptor blockade improves cardiometabolic risk in mouse models of obesity. J Clin Invest 2010;120:2953-66.

44. Cluny NL, Vemuri VK, Chambers AP, et al. A novel peripherally restricted cannabinoid receptor antagonist, AM6545, reduces food intake and body weight, but does not cause malaise, in rodents. $\mathrm{Br} J$ Pharmacol 2010;161:629-42.

45. Costantino CM, Gupta A, Yewdall AW, et al. Cannabinoid receptor 2-mediated attenuation of CXCR4-tropic HIV infection in primary CD4+ T cells. PLoS One 2012;7:e33961.

46. Ramirez SH, Reichenbach NL, Fan S, et al. Attenuation of HIV-1 replication in macrophages by cannabinoid receptor 2 agonists. $J$ Leukoc Biol 2013;93:801-10.

47. $\mathrm{Xu} \mathrm{H}$, Cheng $\mathrm{CL}$, Chen M, et al. Anti-inflammatory property of the cannabinoid receptor-2-selective agonist JWH-133 in a rodent model of autoimmune uveoretinitis. J Leukoc Biol 2007;82:532-41.

48. Zhang M, Adler MW, Abood ME, et al. CB2 receptor activation attenuates microcirculatory dysfunction during cerebral ischemic/ reperfusion injury. Microvasc Res 2009;78:86-94.

49. Condie R, Herring A, Koh WS, et al. Cannabinoid inhibition of adenylate cyclase-mediated signal transduction and interleukin 2 (IL-2) expression in the murine T-cell line, EL4.IL-2. The Journal of biological chemistry 1996;271:13175-83.

50. Massi P, Sacerdote P, Ponti W, et al. Immune function alterations in mice tolerant to delta9-tetrahydrocannabinol: functional and biochemical parameters. J Neuroimmunol 1998;92(1-2):60-6.

51. Molina PE, Amedee AM, LeCapitaine NJ, et al. Modulation of gut-specific mechanisms by chronic $\delta(9)$-tetrahydrocannabinol administration in male rhesus macaques infected with simian immunodeficiency virus: a systems biology analysis. AIDS Res Hum Retroviruses 2014;30:567-78.

52. Lutge EE, Gray A, Siegfried N. The medical use of cannabis for reducing morbidity and mortality in patients with HIV/AIDS. Cochrane Database Syst Rev 2013;4:CD005175.

53. Manuzak JA, Gott TM, Kirkwood JS, et al. Heavy cannabis use associated with reduction in activated and inflammatory immune cell frequencies in antiretroviral therapy-treated human immunodeficiency virus-infected individuals. Clin Infect Dis 2018;66:1872-82.

54. Rizzo MD, Crawford RB, Henriquez JE, et al. HIV-infected cannabis users have lower circulating CD16+ monocytes and IFN- $\gamma$-inducible protein 10 levels compared with nonusing HIV patients. AIDS 2018;32:419-29.

55. Burstein S. Cannabidiol (CBD) and its analogs: a review of their effects on inflammation. Bioorg Med Chem 2015;23:1377-85.

56. Bergamaschi MM, Queiroz RH, Zuardi AW, et al. Safety and side effects of cannabidiol, a Cannabis sativa constituent. Curr Drug Saf 2011;6:237-49.

57. Ribeiro A, Ferraz-de-Paula V, Pinheiro ML, et al. Cannabidiol, a nonpsychotropic plant-derived cannabinoid, decreases inflammation in a murine model of acute lung injury: role for the adenosine $A(2 A)$ receptor. Eur J Pharmacol 2012;678(1-3):78-85.

58. Burstein SH, Zurier RB. Cannabinoids, endocannabinoids, and related analogs in inflammation. Aaps $\mathrm{J}$ 2009;11:109-19.
59. Iuvone T, Esposito G, De Filippis D, et al. Cannabidiol: a promising drug for neurodegenerative disorders? CNS Neurosci Ther 2009;15:65-75

60. Devinsky O, Patel AD, Cross $\mathrm{JH}$, et al. Effect of cannabidiol on drop seizures in the lennox-gastaut syndrome. N Engl J Med 2018;378:1888-97.

61. Sachse-Seeboth C, Pfeil J, Sehrt D, et al. Interindividual variation in the pharmacokinetics of Delta9-tetrahydrocannabinol as related to genetic polymorphisms in CYP2C9. Clin Pharmacol Ther 2009;85:273-6.

62. Jenabian MA, Seddiki N, Yatim A, et al. Regulatory T cells negatively affect IL-2 production of effector T cells through CD39/adenosine pathway in HIV infection. PLoS Pathog 2013;9:e1003319.

63. Nikolova M, Carriere M, Jenabian MA, et al. CD39/adenosine pathway is involved in AIDS progression. PLoS Pathog 2011; $7:$ e1002110.

64. Vandergeeten C, Fromentin R, Merlini E, et al. Cross-clade ultrasensitive PCR-based assays to measure HIV persistence in large-cohort studies. J Virol 2014;88:12385-96.

65. Procopio FA, Fromentin R, Kulpa DA, et al. A novel assay to measure the magnitude of the inducible viral reservoir in HIV-infected individuals. EBioMedicine 2015;2:874-83.

66. Sharkey M, Triques K, Kuritzkes DR, et al. In vivo evidence for instability of episomal human immunodeficiency virus type $1 \mathrm{cDNA} . J$ Virol 2005;79:5203-10.

67. Buzón MJ, Massanella M, Llibre JM, et al. HIV-1 replication and immune dynamics are affected by raltegravir intensification of HAART-suppressed subjects. Nat Med 2010;16:460-5

68. Nowak P, Troseid M, Avershina E, et al. Gut microbiota diversity predicts immune status in HIV-1 infection. AIDS 2015;29:2409-18.

69. Hoel H, Hove-Skovsgaard M, Hov JR, et al. Impact of HIV and Type 2 diabetes on Gut Microbiota Diversity, Tryptophan Catabolism and Endothelial Dysfunction. Sci Rep 2018;8:6725.

70. Lebouché B, Jenabian MA, Singer J, et al. The role of extendedrelease niacin on immune activation and neurocognition in HIVinfected patients treated with antiretroviral therapy - CTN PT006: study protocol for a randomized controlled trial. Trials 2014;15:390.

71. Hill AL, Rosenbloom DI, Goldstein E, et al. Real-time predictions of reservoir size and rebound time during antiretroviral therapy interruption trials for HIV. PLoS Pathog 2016;12:e1005535.

72 . CONSORT -Pilot and Feasibility Trials. http://www.consortstatement.org. (Accessed 19 May 2018).

73. Chan AW, Tetzlaff JM, Altman DG, et al. SPIRIT 2013 statement: defining standard protocol items for clinical trials. Ann Intern Med 2013;158:200-7.

74. International committee of medical journal editors: uniform requirements for manuscripts submitted to biomedical journals: writing and editing for biomedical publication. http://www.icmje.org/ recommendations/archives/2008_urm.pdf.

75. Hileman CO, Funderburg NT. Inflammation, Immune Activation, and Antiretroviral Therapy in HIV. Curr HIVIAIDS Rep 2017;14:93-100.

76. Anderson GD, Chan LN. Pharmacokinetic drug interactions with tobacco, cannabinoids and smoking cessation products. Clin Pharmacokinet 2016;55:1353-68.

77. Kosel BW, Aweeka FT, Benowitz NL, et al. The effects of cannabinoids on the pharmacokinetics of indinavir and nelfinavir. AIDS 2002;16:543-50.

78. Mechoulam R, Hanuš LO, Pertwee R, et al. Early phytocannabinoid chemistry to endocannabinoids and beyond. Nat Rev Neurosci 2014;15:757-64.

79. Portenoy RK, Ganae-Motan ED, Allende S, et al. Nabiximols for opioid-treated cancer patients with poorly-controlled chronic pain: a randomized, placebo-controlled, graded-dose trial. J Pain 2012;13:438-49.

80. Serpell M, Ratcliffe S, Hovorka J, et al. A double-blind, randomized, placebo-controlled, parallel group study of THC/ CBD spray in peripheral neuropathic pain treatment. Eur $J$ Pain 2014;18:999-1012.

81. Pertwee RG. Emerging strategies for exploiting cannabinoid receptor agonists as medicines. Br J Pharmacol 2009;156:397-411.

82. Tramèr MR, Carroll D, Campbell FA, et al. Cannabinoids for control of chemotherapy induced nausea and vomiting: quantitative systematic review. BMJ 2001;323:16-21.

83. Curran HV, Freeman TP, Mokrysz C, et al. Keep off the grass? Cannabis, cognition and addiction. Nat Rev Neurosci 2016;17:293-306.

84. Notcutt W, Langford R, Davies P, et al. A placebo-controlled, parallelgroup, randomized withdrawal study of subjects with symptoms of spasticity due to multiple sclerosis who are receiving long-term Sativex® (nabiximols). Mult Scler 2012;18:219-28. 
85. Wade DT, Makela PM, House H, et al. Long-term use of a cannabisbased medicine in the treatment of spasticity and other symptoms in multiple sclerosis. Mult Scler 2006;12:639-45.

86. Robson P. Abuse potential and psychoactive effects of $\delta$-9tetrahydrocannabinol and cannabidiol oromucosal spray (Sativex), a new cannabinoid medicine. Expert Opin Drug Saf 2011;10:675-85.

87. Pharma GW. SATIVEX-Product Monotgraph, 2015.

88. Zuardi AW, Crippa JA, Hallak JE, et al. Cannabidiol for the treatment of psychosis in Parkinson's disease. J Psychopharmacol 2009;23:979-83.

89. Abbvie Inc. MARINOL-Product Label, 2017.

90. Herkenham M, Lynn AB, Little MD, et al. Cannabinoid receptor localization in brain. Proc Natl Acad Sci U S A 1990;87:1932-6.

91. Boulassel MR, Mercier F, Gilmore N, et al. Immunophenotypic patterns of CD8+ T cell subsets expressing CD8alphaalpha and IL-7Ralpha in viremic, aviremic and slow progressor HIV-1-infected subjects. Clin Immunol 2007;124:149-57.
92. Mercier F, Boulassel MR, Yassine-Diab B, et al. Persistent human immunodeficiency virus- 1 antigenaemia affects the expression of interleukin-7Ralpha on central and effector memory CD4+ and CD8+ T cell subsets. Clin Exp Immunol 2008;152:72-80.

93. Deeks SG, Phillips AN. HIV infection, antiretroviral treatment, ageing, and non-AIDS related morbidity. BMJ 2009;338:a3172.

94. Sparacino CM, Hyldburg PA, Hughes TJ. Chemical and biological analysis of marijuana smoke condensate. NIDA Res Monogr 1990;99:121-40.

95. Noguera-Julian M, Rocafort M, Guillén Y, et al. Gut microbiota linked to sexual preference and HIV infection. EBioMedicine 2016;5:135-46.

96. Monaco CL, Gootenberg DB, Zhao G, et al. Altered virome and bacterial microbiome in human immunodeficiency virus-associated acquired immunodeficiency syndrome. Cell Host Microbe 2016;19:311-22. 